\title{
Artifact Removal from EEG using Spatially Constrained FastICA and Fuzzy Shrink Thresholding Technique
}

\author{
G.Geetha \\ Avinashilingam Institute for Home Science and \\ Higher Education for Women, Research Scholar, \\ Department of Computer Science \\ Coimbatore, Tamilnadu, India
}

\begin{abstract}
This paper presents a novel technique for removing the artifacts from the Electroencephalogram (EEG) signals. EEG signals are influenced by different characteristics, like line interference, EOG (electro-oculogram) and ECG (electrocardiogram). The elimination of artifact from scalp EEGs is of substantial significance for both the automated and visual examination of underlying brainwave actions. These noise sources increase the difficulty in analysing the EEG and obtaining clinical information related to pathology. Hence it is crucial to design a procedure to decrease such artifacts in EEG records. This paper uses Spatially-Constrained Fast ICA (SCFastICA) to separate the Independent Components (ICs) from the initial EEG signal. As the next step, Wavelet Denoising (WD) is applied to extract the brain activity from purged artifacts, where thresholding plays an important role in delineating the artifacts and hence a better thresholding technique called fuzzy Shrink thresholding is applied. Experimental results show that the proposed technique results in better removal of artifacts.
\end{abstract}

\section{General Terms}

Signal Processing, Neural Networks, Soft Computing

\section{Keywords}

Artifact Removal, Electroencephalogram (EEG), Wavelet Denoising, SpatiallyConstrained-FastICA (SC-fastICA)

\section{INTRODUCTION}

The human brain is the most complex organ in the body, and has been a subject of intense study by many researchers from various disciplines. Among the non-invasive methods for probing human brain dynamics, Electroencephalogram (EEG) [15] offers a direct determination of cortical behaviour with millisecond temporal resolution. Electroencephalogram (EEG) is multivariate time series data measured at multiple sensors positioned on the scalp, that imitates electrical prospective produced by the behaviours of brain and is a record of the electrical potentials created by the cerebral cortex nerve cells. There are two categories of EEG, based on

\author{
S.N.Geethalakshmi, PhD. \\ Avinashilingam Institute for Home Science and \\ Higher Education for Women,Associate Professor, \\ Department of Computer Science \\ Coimbatore, Tamilnadu, \\ India
}

where the signal is obtained in the head: scalp or intracranial. Scalp EEG being the focus of the research, is adjunct by small metal discs, also called as electrodes, kept on the scalp with good mechanical and electrical touch. Intracranial EEG is obtained by special electrodes placed in the brain during a surgery. For the purpose of finding the exact voltage of the brain neuron, the electrodes are of low impedance. The variations in the voltage difference among electrodes are sensed and amplified before being transmitted to a computer program. Epileptic seizure $[12,13]$ is an abnormality in EEG gathering and is featured by short and episodic neuronal synchronous discharges with high amplitude. This anomalous synchrony may happen in the brain locally (partial seizures) that is visible only in fewer channels of the EEG signal [14], or including the entire brain (generalized seizures), that is visible in all the channels of the EEG signal.

\section{RELATED WORK}

Han et al., [1] proposed an EEG signal classification technique for epilepsy diagnosis based on AR model and Relevance Vector Machine (RVM). This works includes three phases: Initially, EEG characteristics are gathered from the signals according to AR models, and then these characteristics are analysed. In the next phase, based on the act ofthe characteristics, feature choice was introduced among feature extraction and classifiers. In the last phase, RVM is executed with various AR models, kernel widths, and various subsets ofthe characteristics for the purpose of getting an overview of the technique.

Sukanesh et al., [2] put forth a fuzzy technique and hierarchical aggregation functions decision trees for the classification of epilepsy risk levels from EEG signals [6, 7, 
8]. Statistical spectral feature extraction for classification of epileptic EEG signals is suggested by Seong et al., [3].

Panda et al., [4] given a technique for classification of EEG signal using wavelet transform and Support Vector Machine (SVM) for epileptic seizure diction. Classification of EEG for Epilepsy Diagnosis in Wavelet Domain Using Artificial Neural Network [9] and Multi Linear Regression is suggested by Ercelebi et al., [5].

\section{DATA ACQUISITION}

Current EEG recording equipment is quite smaller and portable than its predecessors, which were cumbersome in nature. The Standard placement guide for electrodes used in EEG measurement is the 10-20 system of electrode placement. The term 10-20 refers to the placement of the electrodes and their relative distances with respect to each other. Each electrode has a letter and a number to identify it. The letters refer to Frontal, Temporal, Central, Parietal and Occipital lobes of the brain. Depending on usage, the number of electrodes may vary. The dataset used for this research work is acquired from Ramakrishna Medical Hospital, Coimbatore. The raw EEG signal consists of 2 sets of data for 160 patients; one corresponding to the pathology and other being normal. Each data set contains 16 channel recordings for 160 patients and the length of the recording is for 10 seconds. The data were sampled at a rate of 256 samples per second. So the total numbers of samples present in the 16 channel recordingfor a single data set are equal to 4096 samples. Therefore the numbers of samples for 160 patients under consideration are 6, 55,360 samples.

\section{METHODOLOGY}

EEG signals are influenced by different characteristics, like line interference, EOG (electro-oculogram) and ECG (electrocardiogram). The elimination of artifact from scalp EEGs is of substantial significance for both the automated and visual examination of underlying brainwave actions. These noise sources increase the difficulty inanalysing the EEG and obtaining clinical information related to pathology. Hence it is crucial to design aprocedure to decrease such artifacts in EEG records.

The architecture of the proposed method for removing the artifacts of EEG data is presented in figure 1.

EEG data implicated is generated based on ICA model as

$x(t)=A s(t)+v(t)(1)$ where $\mathrm{x}(\mathrm{t})=\left[\mathrm{x}_{1}(\mathrm{t}), \mathrm{x}_{2}(\mathrm{t}), \cdots, \mathrm{x}_{\mathrm{M}}(\mathrm{t})\right]^{\mathrm{T}}$ which is a linear mixture of $\mathrm{N}$ sources $\mathrm{s}(\mathrm{t})=\left[\mathrm{s}_{1}(\mathrm{t}), \mathrm{s}_{2}(\mathrm{t}), \cdots, \mathrm{s}_{\mathrm{N}}(\mathrm{t})\right]^{\mathrm{T}}, \mathrm{A}$ is $\mathrm{M} \times \mathrm{N}$ mixing matrix, and $\mathrm{v}(\mathrm{t})=\left[\mathrm{v}_{1}(\mathrm{t}), \mathrm{v}_{2}(\mathrm{t}), \cdots, \mathrm{v}_{\mathrm{M}}(\mathrm{t})\right]^{\mathrm{T}}$ is nothing but the additive noise at the EEG sensors. Here the number of sources is represented as $\mathrm{N}$ and the waveforms are represented as $\mathrm{s}_{\mathrm{i}}(\mathrm{t})$, and mixing matrix $\mathrm{A}$ are all unknown. In order to make the problem simple, the square mixing problem is considered, i.e., $\mathrm{M}=\mathrm{N}$.

\subsection{Preprocessing}

The purpose of conventional filtering is to process raw EEG data $x(t)$ to eliminate $50 \mathrm{~Hz}$ line noise, baseline values, artifacts dwelling in very low frequencies .Here a notch filter is implemented at $50 \mathrm{~Hz}$ to remove line noise ,which is adaptive in nature ,in order to remove noise occurring at $49 \mathrm{~Hz}-51 \mathrm{~Hz}$.

\subsection{Spatially-Constrained ICA (SCICA)}

The main process in the proposed technique is the application of SCICA to obtain artifact ICs from filtered and baseline corrected EEG data $\mathrm{y}(\mathrm{t})$. Here SCICA is described in detail. The key intention is to describe a Spatial Constraint (SC) on the mixing matrix A to symbolize specific prior knowledge or prior assumptions concerning the spatial topography of some source sensor projections, i.e., the SC operates on chosen columns of A and is enforced with reference to a set of predetermined constraint sensor projections, represented by Ac. Thus, the spatially constrained mixing matrix consists of two kinds of columns

$$
A=\left[\widehat{A}_{c}, A_{u}\right]
$$

where $\widehat{A}_{c} \approx A_{c}$ are columns regarded as constraint, and $\mathrm{Au}$ are otherwise regarded as unconstrained columns.

The spatially-constrained ICA technique aims to maximize the statistical independence of the unconstrained sources and at the same time reduce the divergence among the spatially constrained source sensorprojections and their corresponding reference topographies. A deflationary technique is implemented to takeout only desired components, and therefore the output of the spatially-constrained ICA technique is SC-ICs. This results in fast computational time, as all ICs are extracted.

\subsection{Wavelet Denoising (WD) of SC-ICs}

It is significant mentioning that SC-FastICA are expected to correspond to artifacts only; on the other hand, 
some brain action might escape to these gathered signals. As artifacts have a frequency overlap with the brain signals, here conventional filtering technique cannot be utilized, and therefore this paper focuses on using Wavelet Denoising to take away any brain activity from gathered SC-ICs.

The Discrete Wavelet Transform (DWT) examines a finite length time domain signal by breaking up the initial domain in two phases: the detail and approximation data. Consequently, denoising is attained by thresholding the wavelet coefficients FuzzyShrink thresholding method. Finally inverse DWT is utilized to obtain the reconstructed clean signal.

\subsection{Algorithm for FastICA}

1. Choose the number of Independent Components $n$ and set $\mathrm{p}=1$

2. Initialize $\mathrm{W}_{\mathrm{i}}$ randomly

3. Perform Centering and Whitening of data

4. Finding $\mathrm{W}_{\mathrm{i}}$ by non-gaussianity

5. Normalize $\mathrm{W}_{\mathrm{i}}$

6. De-correlate $\mathrm{W}_{\mathrm{i}}$ with the existing $\mathrm{p}-1$ independent components

7. Whether $\mathrm{W}_{\mathrm{i}}$ Converges or not if not go to Step 2

8. Repeat the same for all the $\mathrm{n}$ components

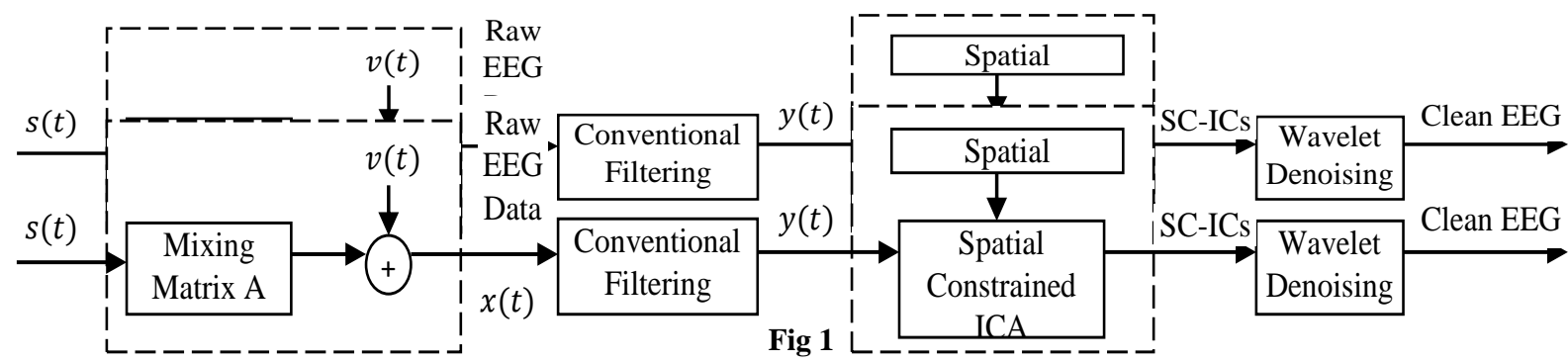

\subsection{Flow chart for Fuzzy Shrink Thresholding}
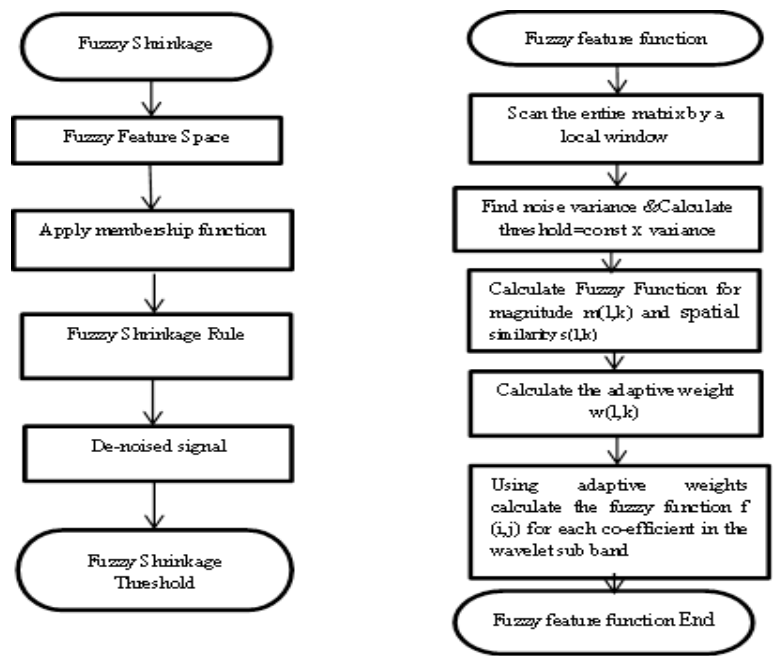

Fig 2
Performance Analysis for Electrical Artifact removal

\begin{tabular}{|l|l|l|l|l|}
\hline \multicolumn{2}{|l|}{ Techniques } & $\begin{array}{l}\text { PSNR } \\
(\mathbf{d B})\end{array}$ & MSE & $\begin{array}{l}\text { Time } \\
\text { Taken } \\
\text { (secs) }\end{array}$ \\
\hline Fast ICA & 42.5436 & 1.0001 & 2.663016 \\
\hline Otsu & 41.8702 & 0.9996 & 1.831568 \\
\hline $\begin{array}{l}\text { Fuzzy } \\
\text { Shrink }\end{array}$ & $\begin{array}{l}\text { S shaped } \\
\text { curve }\end{array}$ & 41.9567 & 0.9799 & 3.115454 \\
\cline { 2 - 5 } & Bsplines & 57.1300 & 0.0322 & 2.760357 \\
\cline { 2 - 5 } & Z shaped & 59.7104 & 0.0178 & 3.003940 \\
\cline { 2 - 5 } & Sigmoid & 47.0331 & 0.3556 & 4.274911 \\
\cline { 2 - 5 } & Triangular & $\mathbf{6 0 . 7 9 9 9}$ & $\mathbf{0 . 0 3 1 8}$ & $\mathbf{3 . 3 4 9 1 8 4}$ \\
\cline { 2 - 5 } & Bell curve & 51.0188 & 0.1337 & 4.228277 \\
\cline { 2 - 5 } & $\begin{array}{l}\text { Gaussian } \\
\text { curve }\end{array}$ & 51.5807 & 0.1193 & 5.064617 \\
\hline
\end{tabular}

\section{EXPERIMENTAL RESULTS}

This section presents the evaluation of the proposed artifact removal technique 
Performance Analysis for EyeBall Movement Artifact removal

\begin{tabular}{|c|c|c|c|c|}
\hline \multicolumn{2}{|c|}{ Techniques } & $\begin{array}{c}\text { PSNR } \\
\text { (dB) }\end{array}$ & MSE & $\begin{array}{c}\text { Time } \\
\text { Taken } \\
\text { (secs) }\end{array}$ \\
\hline \multicolumn{2}{|c|}{ Fast ICA } & 44.6751 & 1.0007 & 1.481553 \\
\hline \multicolumn{2}{|c|}{ Otsu } & 45.0538 & 1.0008 & 1.372437 \\
\hline \multirow[t]{7}{*}{$\begin{array}{l}\text { Fuzzy } \\
\text { Shrink }\end{array}$} & $\begin{array}{l}\text { S shaped } \\
\text { curve }\end{array}$ & 44.7405 & 0.9931 & 2.581424 \\
\hline & Bsplines & 59.9467 & 0.0310 & 2.334827 \\
\hline & $\mathrm{Z}$ shaped & 67.5135 & 0.0054 & 2.713516 \\
\hline & Sigmoid & 48.9706 & 0.4016 & 3.690825 \\
\hline & Triangular & 65.1513 & 0.0037 & 2.976519 \\
\hline & Bell curve & 55.3662 & 0.0900 & 4.035033 \\
\hline & $\begin{array}{c}\text { Gaussian } \\
\text { curve }\end{array}$ & 57.9451 & 0.0492 & 4.074172 \\
\hline
\end{tabular}

Performance Analysis for Eye Blink Artifact removal

\begin{tabular}{|c|c|c|c|c|}
\hline \multicolumn{2}{|c|}{ Techniques } & PSNR (dB) & MSE & $\begin{array}{l}\text { Time } \\
\text { Taken } \\
\text { (secs) }\end{array}$ \\
\hline \multicolumn{2}{|c|}{ Fast ICA } & 46.9401 & 1.0015 & 1.374718 \\
\hline \multicolumn{2}{|l|}{ Otsu } & 47.3033 & 1.0015 & 1.381986 \\
\hline \multirow{7}{*}{$\begin{array}{l}\text { Fuzzy } \\
\text { Shrink }\end{array}$} & $\begin{array}{l}\text { S shaped } \\
\text { curve }\end{array}$ & 47.0988 & 0.9699 & 2.566778 \\
\hline & Bsplines & 62.2505 & 0.0307 & 2.394129 \\
\hline & $\mathrm{Z}$ shaped & 62.6926 & 0.6277 & 2.795805 \\
\hline & Sigmoid & 50.9111 & 0.4068 & 3.427329 \\
\hline & Triangular & 65.8221 & 0.0135 & 2.920010 \\
\hline & Bell curve & 56.8389 & 0.1095 & 2.719094 \\
\hline & \begin{tabular}{|l|}
$\begin{array}{l}\text { Gaussian } \\
\text { curve }\end{array}$ \\
\end{tabular} & 57.3282 & 0.0979 & 3.244250 \\
\hline
\end{tabular}

Performance Analysis for Spit Swallowing Artifact removal

\begin{tabular}{|c|c|c|c|c|}
\hline \multicolumn{2}{|c|}{ Techniques } & $\begin{array}{l}\text { PSNR } \\
\text { (dB) }\end{array}$ & MSE & $\begin{array}{l}\text { Time } \\
\text { Taken } \\
\text { (secs) }\end{array}$ \\
\hline \multicolumn{2}{|c|}{ Fast ICA } & 49.5212 & 1.0036 & 1.432256 \\
\hline \multicolumn{2}{|l|}{ Otsu } & 49.2044 & 1.0031 & 1.392559 \\
\hline \multirow{7}{*}{$\begin{array}{l}\text { Fuzzy } \\
\text { Shrink }\end{array}$} & $\begin{array}{l}S \text { shaped } \\
\text { curve }\end{array}$ & 49.3315 & 0.9742 & 2.564763 \\
\hline & Bsplines & 62.9732 & 0.0436 & 2.404310 \\
\hline & $\mathrm{Z}$ shaped & 64.3253 & 0.0319 & 2.706861 \\
\hline & Sigmoid & 52.4775 & 0.4754 & 3.391901 \\
\hline & Triangular & 65.5235 & 0.0242 & 3.022603 \\
\hline & Bell curve & 58.7064 & 0.1172 & 3.282242 \\
\hline & $\begin{array}{l}\text { Gaussian } \\
\text { curve }\end{array}$ & 58.6816 & 9.1171 & 2.882498 \\
\hline
\end{tabular}

Performance Analysis for Jaw Clenching Artifact removal

\begin{tabular}{|c|c|c|c|c|}
\hline \multicolumn{2}{|c|}{ Techniques } & PSNR (dB) & MSE & $\begin{array}{c}\text { Time } \\
\text { Taken } \\
\text { (secs) }\end{array}$ \\
\hline \multicolumn{2}{|l|}{ Fast ICA } & 49.5212 & 1.0036 & 1.432256 \\
\hline \multicolumn{2}{|l|}{ Otsu } & 49.2044 & 1.0031 & 1.392559 \\
\hline \multirow{7}{*}{$\begin{array}{l}\text { Fuzzy } \\
\text { Shrink }\end{array}$} & $\begin{array}{l}\text { S shaped } \\
\text { curve }\end{array}$ & 49.3315 & 0.9742 & 2.564763 \\
\hline & Bsplines & 62.9732 & 0.0436 & 2.404310 \\
\hline & Z shaped & 64.3253 & 0.0319 & 2.706861 \\
\hline & Sigmoid & 52.4775 & 0.4754 & 3.391901 \\
\hline & Triangular & 65.5235 & 0.0242 & 3.022603 \\
\hline & Bell curve & 58.7064 & 0.1172 & 3.282242 \\
\hline & $\begin{array}{l}\text { Gaussian } \\
\text { curve }\end{array}$ & 58.6816 & 9.1171 & 2.882498 \\
\hline
\end{tabular}

For all the five types of Artifact considered the Fuzzy Shrink thresholding performs better than otsu. Above all triangular membership performs well when compared to the other six membership function Subsections 


\section{CONCLUSION}

This paper focuses on removing the artifacts from Electroencephalogram (EEG) signals. Artifact removal is an important process before analysing the EEG signal for prediction of any pathological diseases. Various researchers have focused on this process and developed their own technique for artifact removal. This paper intends on developing a new technique to remove the artifact from EEG. The proposed approach uses Spatially Constrained Fast ICA (FastICA) to separate the exact Independent Components (ICs) from the initial EEG signal. Then, Wavelet Denoising is applied to extract the brain activities from purged artifacts. The thresholding technique used in this paper is Fuzzy Shrink thresholding which is compared with Otsu thresholding.

\section{ACKNOWLEDGMENTS}

Our thanks to the experts who have contributed towards development of the template.

\section{REFERENCES}

[1] Shi-Yun Shao, Kai-Quan Shen, Chong Jin Ong, WilderSmith, E and Xiao-Ping Li, "Automatic EEG Artifact Removal: A Weighted Support Vector Machine Approach with Error Correction", IEEE Transactions on Biomedical Engineering, Vol. 56, Pp. 336-344, 2009.

[2] Shi-Yun Shao, Kai-Quan Shen, Chong-Jin Ong, XiaoPing $\mathrm{Li}$ and Wilder-Smith, E.P.V, "Automatic identification and removal of artifacts in EEG using a probabilistic multi-class SVM approach with error correction", IEEE International Conference on Systems, Man and Cybernetics, Pp. 1134-1139, 2008.

[3] Kavitha, P.T, Lau, C.T and Premkumar, A.B., "Modified ocular artifact removal technique from EEG by adaptive filtering", International Conference on Information, Communications \& Signal Processing, Pp. 1-5, 2007.

[4] Kyung Hwan Kim, Hyo Woon Yoon and Hyun Wook Park, "Improved algorithm for ballistocardiac artifact removal from EEG simultaneously recorded with fMRI", 26th Annual International Conference of the IEEE Engineering in Medicine and Biology Society, Vol.1, Pp. 936-939, 2004.

[5] P. LeVan, E. Urrestarrazu, and J. Gotman, "A system for automatic artifact removal in ictal scalp EEG based on independent component analysis and Bayesian classification“, Clinical Neurophysiology, Vol. 117, No.4, pp. 912- 927, 2006.

[6] R.J. Croft and R.J. Barry, "Removal of ocular artifact from the EEG: areview ", Clinical Neurophysiology, Vol. 30, No.1, pp. 5- 19, 2000.

[7] CA.Joyce, IF.Gorodnitsky, M.Kutas, "Automatic removal of eye movement and blink artifacts from EEG data using blind component separation “,Phychophysiology., Vol. 41, No.2, pp.313- 325, 2004.

[8] V. Krishnaveni, S. Jayaraman, S. Aravind, V. Hariharasudhan, K.Ramadoss, "Automatic identification and Removal of ocular artifacts from EEG using Wavelet transform ", Measurement Science Review, Vol. 6, No.4, pp.45-57, 2006.
[9] V. Krishnaveni, S. Jayaraman, N. Malmurugan, A. Kandasamy, D. Ramadoss, "Non adaptive thresholding methods for correcting ocularartifacts in EEG ", Academic Open Internet Journal, Vol.13, 2004.

[10] Shlomit Yuval-Greenberg, Orr Tomer, Alon S. Keren, Israel Nelken and Leon Y. Deouell, "Transient Induced Gamma-Band Response in EEG as a Manifestation of Miniature Saccades“, Neuron, Vol.58, No.3, pp.429441, 2008.

[11] S. Verobyov and A. Cichocki. Blind noise reduction of multisensory signals using ICA and subspace filtering, with application to EEG analysis. Biological Cybernetics, 86:293-303, 2002.

[12] M. Potter, N. Gadhok, and W. Kinsner. Separation performance of ICA on simulated EEG and ECG signals contaminated by noise. Canadian Journal of Electrical and Computer Engineering, 27(3):123-127, July 2002.

[13] S. Choi, A. Cichocki, H. Park, S. Lee, blind Source "Separation and Independent Component Analysis : A Review", Neural Information Processing - Letters and Reviews, Vol. 6, no. 1, January 2005.

[14] A. Cichocki, Shun-ichi Amari, Adaptative blind Signal and Image Processing Learning Algorithms and Applications, John Wiley \& Sons, ltd, 2002.

[15] Sutherland, M.T., and Tang A.C. " Blind source separation can recover systematically distributed neuronal sources from "resting" EEG", Proceedings of the Second International Symposium on Communications, Control, and Signal Processing (ISCCSP 2006), Marrakech, Morocco, March 13-15.

[16] Joep J. M. Kierkels, Geert J. M. Van Botel, and Leo L. M. Vogten. 'A Model-Based Objective Evaluation of Eye Movement Correction in EEG Recordings", IEEE Transactions on biomedical engineering, vol. 53, No. 2, February 2006.

[17] Muhammad Tahir Akhtar and Christopher J. James, "Focal Artifact Removal from Ongoing EEG - A Hybrid Approach Based on Spatially-Constrained ICA and Wavelet De-noising", Annual International Conference of the IEEE EMBSMinneapolis, Pp. 4027-4030, 2009.

[18] N.P. Castellanos and V.A. Makarov, "Recovering EEG brain signals: Artifact suppression with wavelet enhanced independent componentanalysis," J. Neuroscience Methods, vol. 158, pp. 300-312, 2006.

[19]G.Geetha ， Dr.S.N.Geethalakshmi,"Artifact Removal from EEG using Spatially Constrained Independent Component Analysis and Wavelet Denoising with Otsu' Thresholding Technique,” Elsevier Proceedings,2011.

[20]Jamal Saeedi, Mohammad Hassan Moradi, Karim Faez”A new wavelet-based fuzzy single and multi-channel image denoising",Elsevier Image and vision Computing,pp.1611-1623,2010. 